\title{
STUDIES ON THE HIGH MOLECULAR SUBSTANCES IN SAKÉ
}

\author{
PART 1. SPECIAL PRODUCTS OF ASPERGILLUS \\ FUNGI IN CONCERN TO THE OCCURRENCE \\ OF THE “PROTEIN TURBIDITY” \\ IN SAKÉ
}

TADAHIKO ANDO, YONOSUKE IKEDA, KISETSU SHUZUI, and SHIGEJI IIDA

\author{
The Scientific Research Institute Ltd., and the Institute of Applied \\ Microbiology, University of Tokyo
}

Received for publication July 1, 1957

Saké is a representative alcoholic beverage widely consumed in Japan, brewed from rice, and contains various kinds of metabolic products of Aspergillus oryzae and Saccharomyces cerevisiae. In the meantime, because rice is the staple food for the Japanese, during the time of the first war, it was strongly desired to produce saké from raw materials other than rice. Thenceforth, strenous efforts have been put forth to investigate the chemical constituents of natural saké and to synthesize an imitation from knownchemicals. But as it is readily understood, it was not so easy to produce such an imitation artificially, and after successive efforts continuing for a period of more than thirty years the idea has been partially realized, because, at the present time, artificial saké is very much similar in quality to natural saké and is getting great reputation on the market. The output of artificial saké in the 1955 fiscal year is estimated to be ca. 35 million gallon, about one-fourth of that of natural saké.

The present authors do not intend to describe the history of its improvement in this paper, but rather wish to point out one of the major differences which still exist between synthetic saké and natural saké. In other words, with the view that the main dfference existing between the two kinds of saké might be caused by the presence or absence of high moleclar components, the isolation of high moleclar substances from natural saké by electrophoretic means and the measurement of their amounts contained in saké by immunological means were attempted. Among the four substances so far studied, two mucoprotein-like substances produced exclusively by Aspergillus fungi and closely related to the "protein turbidity" phenomenon in saké will be described here.

\section{EXPERIMENTAL}

The importance of high molecular components for saké taste 
Preliminarily, the responsibility of the high molecular fraction in natural saké upon the taste of saké was evaluated by the following way.

\section{Preparation of high molecular fraction by dialysis}

First, 1,800 $\mathrm{ml}$ best grade saké (trademark "Masumi ") was concentrated to one third of its volume by a flash evaporator, and after pouring the con-

Table 1. Chemical Composition of Natural and Artificial Saké

\begin{tabular}{l|c|c}
\multicolumn{1}{c|}{ Constituents } & Natural Saké & Artificial Saké* \\
\cline { 1 - 2 } Alcohol & $16 \%$ & $16 \%$ \\
Total acid & $0.10 \%$ & $0.08 \%$ \\
(as succinic acid) & $0.11 \%$ & $0.05 \%$ \\
$\begin{array}{l}\text { Amino acid } \\
\text { (as glycine) }\end{array}$ & $3.19 \%$ & $3.58 \%$ \\
$\begin{array}{l}\text { Direct reducing } \\
\text { sugar (as glucose) } \\
\text { Total sugar }\end{array}$ & $3.78 \%$ & $3.90 \%$ \\
(as glucose) & 4.2 & 3.9 \\
pH & & \\
\hline
\end{tabular}

* In Regard of the definition of artificial and synthetic saké, refer to the footnote of Table 2 .

centrate into a cellophane sac, it was stood in running water for dialysis. After the elapse of 24 hours, the contents of the sac were concentrated to $30 \mathrm{ml}$ in vacuo. The materials of this syrup were almost insoluble in $70 \%$ alcohol and reacted strongly $(+++)$ with $\alpha$-naphthol, weakly $(+)$ with biuret reagent, and faintly $( \pm)$ with ninhydrin and p-anisidine.

2. The quality of synthetic saké supplemented with the high molecular fraction of natural saké.

Table 2. Composition of Basal Liquor (Pure Synthetic Saké*)

\begin{tabular}{l|c}
\multicolumn{1}{c|}{ Constituents } & Content $(\%)$ \\
\hline Alcohol & 15.5 \\
Glucose & 3.05 \\
Liquid dextrin & 0.55 \\
Sodium glutamate & 0.015 \\
L-Alanine & 0.010 \\
L-Glycine & 0.005 \\
Succinic acid & 0.0084 \\
Sodium succinate & 0.012 \\
Lactic acid & 0.012 \\
KH $_{2} \mathrm{PO}_{4}$ & 0.006 \\
$\mathrm{CaHPO}_{4}$ & 0.006 \\
$\mathrm{NaCl}$ & 0.010
\end{tabular}

* The general name for artificial saké is "Gôseishu" (synthetic saké) or "Shin-seishu" (new-fashion saké), but in this paper, the kind of saké which is synthesized from known-chemicals will be called synthetic saké. Artificial saké on the market is manufactured by treating synthetic saké with enzymes or by the supplement of a small amount of natural substances such as rice koji (rice plus $A s p$. oryzae), or soy bean koji (soy bean-protein plus $A s p$. oryzae). 
For the purpose to evaluate the role of the high molecular fraction in saké taste, $3 \mathrm{ml}$ of the above mentioned syrup was added to $180 \mathrm{ml}$ basal liquor, so that the resulting liquor might contain an equal amount of high molecular fraction as the original natural saké. In this experiment, the most simple form of synthetic sake which is shown in Table 2 was employed as the basal liquor. Upon criticism two days afterwards given by several technicians, it was commented that the quality had been remarkably improved by the addition of the high molecular fraction of natural saké.

\section{Separation of D-1 and D-2 substances by electrophoretic technique}

As preliminary work suggested that the components in the high molecular fraction might be essential for the palatability of saké, extensive attempts to separate them by electrophoretic means were carried out.

\section{Detection of bromophenol blue positive substances on the paper}

The kind of high molecular fraction prepared just the same as that mentioned above but dialysed for a longer time ( 72 hours) than the case was spotted $(0.05-0.10 \mathrm{ml})$ on the centre of a paper strip. Employing the paper electrophoretic apparatus which had been illustrated previously(1), electromigration was carried out under the following conditions: phosphate buffer $0.05 \mathrm{~m}, \mathrm{pH} 7.6$; potential gradient 400 D. C. volt, $0.5 \mathrm{~mA} / \mathrm{cm}$; migration period, 5 hours at room temperature. When migration was completed, the paper was removed from the apparatus and sprayed with five different reagents. Among these, bromophenol blue (B.P.B.) was found to be most suitable for the detection of the materials on the paper( ${ }^{(2)}$. The staining was performed as follows: after letting the paper stand for 15 minutes in alcoholic solution containing $1 \%$ B. P. B. and being satulated with bichloride of mercury, it was washed several times with $1 \%$ acetic acid solution, and subsequently exposed to ammonia gas. As a result, four blue spots appeared on the paper as seen in Figure $3 \mathrm{~A}$. Besides B. P. B., $\alpha$-naphthol reacted strongly with the materials, but for technical reasons the B. P. B. staining was employed as the standard method throughout this work.

\section{Separation of $D-1$ and $D-2$ substances by zone electrophoretic technique}

In this experiment, the high molecular fraction was purified further before allowing it to serve for electrophoretic analysis. As seen in Figure 1 , the frash-evaporated fraction corresponding to $1,800 \mathrm{ml}$ natural saké was dialysed for 72 hours, and then the high molecular substances of the sac were precipitated by the addition of ethylalcohol to the concentration of 65 volume per cent. The precipitate, upon being washed with alcohol and dried up, gave $340 \mathrm{mg}$ of a slightly grey colored powder. This powder will hereafter be referred to as D-complex. From the supernatant, $440 \mathrm{mg}$ of a slightly yellow colored power was recovered, but no further study was 
Figure 1. Preparation of D-complex by Dialysis and Fractional Precipitation

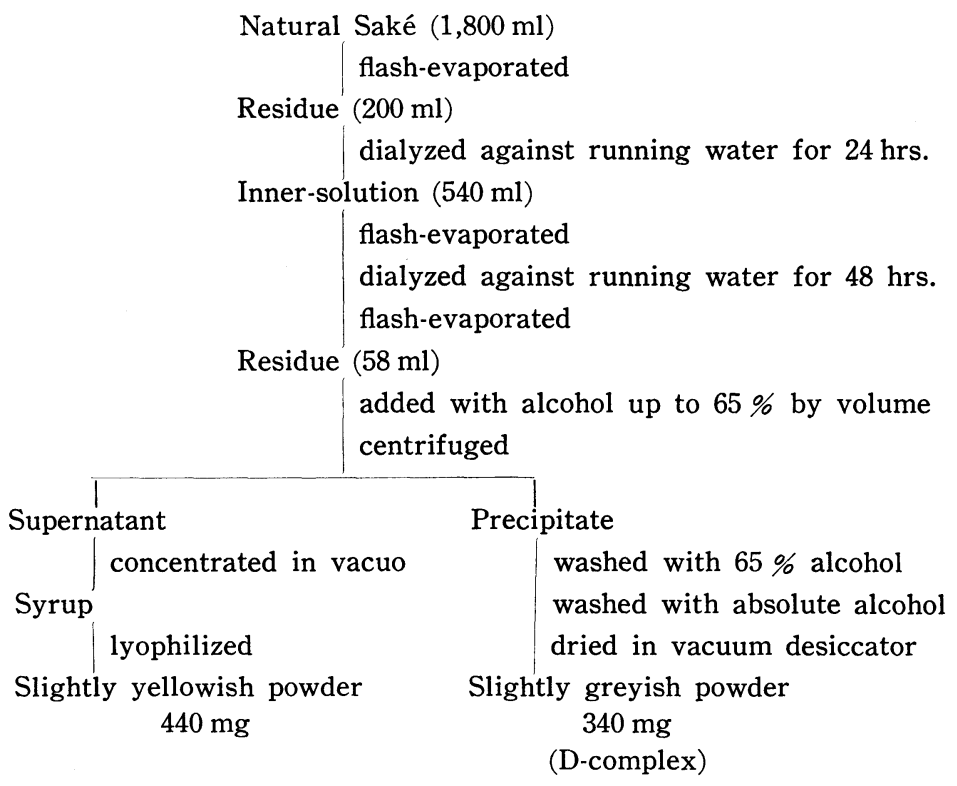

attempted upon it because this fraction did contain a very small amount of B. P. B. positive substances.

The apparatus employed for the separation is illustrated in Figure 2. Prior to operation, the vessel, $45 \times 5 \times 2 \mathrm{~cm}^{3}$ in volume and made of synthetic resin, was filled up to the depth of $10 \mathrm{~mm}$ with about $150 \mathrm{~g}$ potato starch, and excess buffer solution $(\mathrm{m} / 50, \mathrm{pH} 7.7$, in which the starch was suspended) was overflowed by decantation. The sample D-complex (about $50 \mathrm{mg}$ ) was dissolved in a small smount of buffer solution and, after mixing with buffered starch, was dropt into a hole digged on the centre of the starch

Figure 2. Zone Electrophoretic Apparatus

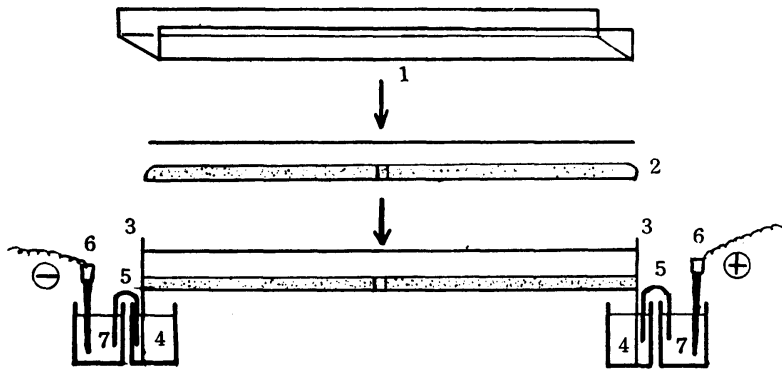

1 Zone electrophoretic vessel

2 Buffered starch

3 Supporting board

4 Buffer vessel
$51 \mathrm{M} \mathrm{KCl} 3 \%$ agar bridge

6 Carbon electrode

7 Electrode vessel 
belt. When the setting had completed, the electric current was induced. Electromigration was carried out at room temperature for eight hours in phosphate buffer $(\mathbf{M} / 50, \mathrm{pH} 7.8)$, at a potential gradient of $400 \mathrm{D}$. C. volt and at a current of $10-15 \mathrm{~mA}$.

The site of B. P. B. positive substances after migration were detected in the following way: A paper strip was placed in contact with the starch belt for a few minutes, and when printing was completed, the paper was treated by B. P. B. reagent as described above. The paper thus treated gave two blue bands as seen in Figure $3 \mathrm{~B}$, and these responsible substances

Figure 3. Electrophoretic Behavior of B. P. B. Positive Substances

A. On a paper electrophoretic apparatus (The sample was prepared by dialysis for 24 hours)

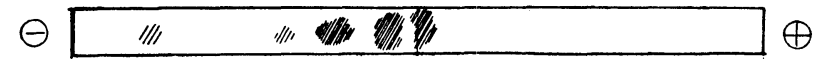

B. On a zone electrophoretic apparatus (The sample was prepared by dialysis for 72 hours and sequential fractional precipitation*)

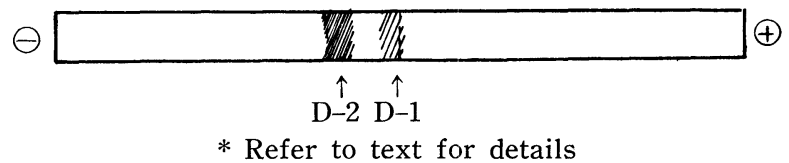

in the bands were named D-1 $(0.5 \mathrm{~cm}$ towards cathode) and $\mathrm{D}-2(2.4 \mathrm{~cm}$ towards cathode). The spots corresponding to the third and fourth substances shown in Figure 3-A was not detected in this experiment because the sample was purified in advance through fractional precipitation.

Both substances, D-1 and D-2, were extracted with water from the corresponding zone on the starch belt, and after being neutralized with dil. $\mathrm{HCl}$ solution precipitated by the addition of ethylalcohol. Powder thus obtained was either white or greyish, and the yield obtained from the original saké was calculated as $0.0005 \%$ for D-1 and $0.0025 \%$ for D-2.

\section{Chemical constituents of D-1 and D-2 substances}

When a sufficient amount of D-1 and D-2 substances were stocked by repetation of the process mentioned above, they could be served for chemical analysis.

\section{Purity of $D-1$ and $D-2$ substances}

In order to find the extent of the samples purified by the process, they were further analysed by a Tieselius type electrophoretic apparatus (manufactured by Hitachi Co.). Two samples, D-1, and D-2, were dissolved separately in $5 \mathrm{ml}$ of $0.05 \mathrm{~m}$ phospate buffer ( $\mathrm{pH} \mathrm{7.8)}$ and their migration pattern in the apparatus was photographed mechanically. The electromigration was performed at a potential gradient of $80 \mathrm{D}$. C. volt and at a current of 


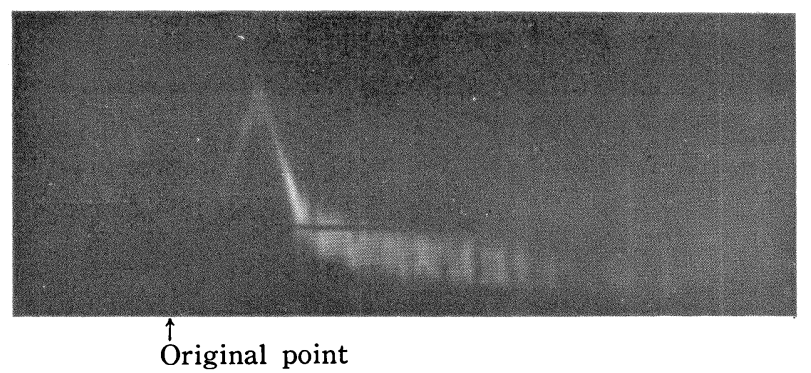

Figure 4. Migration Pattern of D-2 Substance on a Tieselius

Type Electrophoretic Apparatus

$8 \mathrm{~mA}$. As the photograph in Figure 4 illustrate, it was found that the figure corresponding to D-2 showed only one peak after the elapse of 60 minutes, indicating that the separation by the zone electrophoretic technique was almost complete. Though D-1 substance gave a figure similar to that of $\mathrm{D}-2$, there was a little difference in mobility. It is regrettable that the complete figure of D-1 is not presented here because of it being damaged too severly.

\section{Color tests and nitrogen content}

Several kinds of color tests were attempted upon D-1 and D-2 substances, with the result shown in Table 3. Among them, Molishe's $\alpha$-naph-

Table 3. Color Tests and Elemental Analysis (D-1 and D-2 substances)

\begin{tabular}{l|c|c}
\hline & D-1 & D-2 \\
\hline Biuret reaction & \pm & + \\
Ninhydrin reaction & \pm & \pm \\
Folin's reaction & - & \pm \\
$\alpha$-Naphthol reaction & +++ & +++ \\
Ultraviolet light absorption & $275 \mathrm{~m} \mu$ & $275 \mathrm{~m} \mu$ \\
C content \% & 39.3 & 41.0 \\
H content \% & 6.1 & 6.2 \\
N content $\%$ & 4.9 & 2.8 \\
\hline
\end{tabular}

thol reaction was most positive, so this result was taken as an evidence supporting the view that the major part of the substances might be consisted of polysaccharides. In addition, both of the substances showed maximal absorption at $275 \mathrm{~m} \mu$ when tested on an electrospectrophotometer. With referential purposes, a simple elemental analysis was run with desiccated samples. As seen in Table 3, a higher content of $\mathrm{N}$ in sample $\mathrm{D}-1$ appeared to be characteristic.

\section{Hydrolyzed products}

Amino acid composition of the samples was analysed as follows: The 
VoL. 3

samples were decomposed with $6 \mathrm{~N} \mathrm{HCl}$ for 20 hours, and after that, hydrochloric acid was removed in vacuo. Paper chromatography was performed in the two directional system, by employing $n$-butanol-acetic acid-water (4: $1: 1)$ and ammoniacal phenol $(0.15 \mathrm{gm}$ in $100 \mathrm{ml}$ of $70 \%$ phenol) as the solvents, and ninhydrin $(0.1 \%)$ as the developing agent. The kinds of amino acids detected by this method are listed in Table 4. As seen in the

Table 4. Hydrolysed Products (D)-1 and D-2 substances)

\begin{tabular}{l|l|l}
\hline & \multicolumn{1}{|c|}{ D-1 } & \multicolumn{1}{|c}{$\mathrm{D}-2$} \\
\hline & Leucine & Leucine \\
& Valine & Valine \\
& Alanine & Alanine \\
Amino acids & Glycine & Glycine \\
& Serine & Serine \\
& Glutamic acid & Glutamic acid \\
& Aspartic acid & Aspartic acid \\
& Two unidentified spots & Methionine \\
\hline \multirow{3}{*}{ Sugars } & Mannose & Mannose \\
& Galactose & Galactose \\
& Glucose (trace) & \\
\hline & Snidentified uronic-like & \\
\hline
\end{tabular}

table, no significant difference was observed between $\mathrm{D}-1$ and $\mathrm{D}-2$, though this does not mean that no other amino acid except those listed are contained in the samples.

In order to find out the sugar composition of the presumed polysaccharides, the sample were hydrolyzed with $1 \mathrm{~N} \mathrm{HCl}$ for two hours. Paperchromatography was performed employing the multiple ascending system, by using $n$-butanol-pyridine-water $(3: 2: 1.5)$ as the solvent and ammoniacal silver nitrate $(5 \%)$ and $p$-anisidine as the developing reagents. As the results in Table 4 show, among the two samples mannose and galactose were detected in common, but glucose and an unidentified uronic-like substance were detected in addition in D-1.

By summarizing data so far obtained, it might be reasonable to state here that D-1 and D-2 are sorts of mucoprotein consisting of protein and polysaccharides.

\section{Measurement of D-complex by serological means}

In order to measure the amount of D-1 and D-2 substances contained in natural saké, a serological method was established in the following way.

\section{Preparation of antisera}

As it was hard to prepare D-1 substance in the amount sufficient to 
let it serve for the preparation of antiserum, D-complex and D-2 substance were employed instead. These substances were injected intravenously into rabbits in an amount from 5 to $10 \mathrm{mg}$ in each injection, at an interval from one to two weeks. Perfect immunization was attained after 113 days in the case of D-complex and 49 days in the case of D-2 substance, and the blood was stocked in a refrigerator with the addition of $0.02 \%$ marzonin after the treatment at $56^{\circ} \mathrm{C}$ for 30 minutes.

\section{Precipitation tests between the antisera and the samples}

The antigen-antibody precipitation test was performed in two ways, by using the one-dimensional diffusion method as a routine technique and the mixed method in special cases.

In the one-dimensional diffusion method, $0.3 \mathrm{ml}$ antiserum was taken in a small test tube ( $3 \mathrm{~mm}$ in diameter) and the above antigen, which had been diluted with $0.15 \mathrm{~m} \mathrm{NaCl}$ solution to an appropriate concentration, was superposed quietly. After placing the test tubes in an incubator at $37^{\circ}$ for 30 or 60 minutes, those tubes developing a white ring in the contact surface of the two liquids were considered as "reaction positive." The term positive implies that the testing sample, such as D-complex, D-2, or natural saké, contains enough amount of antigen which reacts with the antibody in the serum. As the unit of antigen density in a sample, the maximal dilution ratio giving a positive reaction with the antiserum was adopted. When the dilution of $1: 64,000$ was attained the titre was recorded with 64,000 . It is needless to say that the absence of positive reaction between the antiserum and $\mathrm{NaCl}$ solution, between normal serum and $\mathrm{NaCl}$ solution, and between normal serum and the testing solution was checked at the same time.

In the mixing method, an amount of $0.5 \mathrm{ml}$ of antiserum was reacted with $0.5 \mathrm{ml}$ of diluted samples in small test tubes $(7 \mathrm{~mm}$ in diameter) and the highest dilution ratio giving a positive reaction was recorded in the way described above.

By depending mainly upon the one-dimensional diffusion method, the antigen activity of the D-complex and D-2 substance was measured. As

Table 5. Precipitation Tests between Antisera and D-complex

\begin{tabular}{l|c|c}
\hline \multirow{2}{*}{ Antigens } & \multicolumn{2}{|c}{ The maximal dilution ratio of the antigens } \\
\cline { 2 - 3 } & Anti-D-serum & Anti-D-2-serum \\
\hline D-complex & $1: 64,000$ & $1: 32,000$ \\
D-1 & $1: 15,000$ & $1: 32,000$ \\
D-2 & $1: 32,000$ & $1: 32,000$ \\
Yeast gum* & $0 * *$ & 0 \\
Koji mold gum* & 0 & 0 \\
* Of these substances, details will appear elsewhere. \\
** No positive reaction was observed even at $\times 400$ dilution.
\end{tabular}


the results shown in Table 5 indicate, both anti-D-serum and anti-D-2-serum cross-reacted with D-complex as well as with D-2 substance, but no positive reaction was observed with yeast gum and koji mold gum.

In regard to this, immunological defference between two antisera was studied extensively. A quantity of $0.5 \mathrm{ml}$ anti-D-serum was reacted with 0.5 $\mathrm{ml}$ diluted antigen-D-2, and the test tubes showing most remarkable reaction with $\mathrm{D}-2$ were centrifuged after they were placed for four hours at $37^{\circ} \mathrm{C}$ and overnight in a refrigerator. When the supernatant was tested serologically with D-1 and D-2 antisera, no precipitation occurred, whereas in the original solution precipitation was caused by the addition. The result of this absorption test indicates that the two mucoproteins, D-1 and D-2, are serologically so closely related that it is imposible to measure their amount separately by immunological means.

\section{Measurement of D-complex in natural and artificial saké}

Natural and artificial sake available on the market were employed as the antigen. After removal of the alcohol from sake in vacuo, the residue was diluted with $0.15 \mathrm{~m} \mathrm{NaCl}$ solution to an appropriate concentration to let it react with anti-D or anti-D-2 serum. The test was performed by the one dimensional diffusion method and the amount of antigen corresponding to D-complex or D-2 in the original saké was calculated as follows :

$$
S=\frac{1}{a} \times b \times 100 \text { (per cent) }
$$

Where, in the equation $a$ indicates the highest dilution value of $\mathrm{D}$-complex

Table 6. The Contents of D-equivalent Substances Contained in Natural and Artificial Saké

\begin{tabular}{|c|c|c|}
\hline \multirow{2}{*}{ Samples } & \multicolumn{2}{|c|}{ “D” contents (per cent) } \\
\hline & by " Anti-D", & by "Anti-D-2", \\
\hline 1 (Natural saké " super-blend") & 0.0125 & 0.0125 \\
\hline 2 (Natural saké “ first” grade) & 0.0125 & 0.0062 \\
\hline 3 (Natural saké “ first” grade) & 0.0125 & 0.0125 \\
\hline 4 (Natural saké “ second" grade) & 0.0125 & 0.0125 \\
\hline 5 (Natural saké “ first” grade) & 0.0125 & 0.0125 \\
\hline 6 (Natural saké “ first” grade) & 0.0062 & 0.0062 \\
\hline 7 (Natural saké “ super-blend”) & 0.0031 & 0.0031 \\
\hline 8 (Natural saké “ first” grade) & 0.0031 & 0.0031 \\
\hline 9 (Natural saké “ second" grade) & 0.0031 & 0.0031 \\
\hline 10 (Artifical sakeé “ second" grade) & 0.0016 & 0.0016 \\
\hline 11 (Artificial saké " second" grade) & 0.0016 & 0.0016 \\
\hline 12 (Pure synthetic saké) & 0.0000 & 0.0000 \\
\hline
\end{tabular}

The trademarks of the samples were : Masumi, Kitanoi, Ryozeki, Sakura-masamune, Ohzeki, Fukumusume, Gekkeikan, Akinishiki, Tentaka, Shinshin and Rikyu. 
$(64,000)$ or D-2 $(32,000)$ and $b$ the highest dilution value of the sample.

As a result, it was found out that the amount of D-complex contained in natural saké was more larger than in artificial saké, though considerable deviation was observed (Table 6). The reason why the antiserum reacted positively with artificial saké may be explained that the artificial saké was prepared from synthetic saké and 5-10\% koji (a complex of rice and mold).

\section{Relationship between the content of D-complex in natural saké and the "protein turbidity"}

When the experiments proceeded to this step, the authors came to recognize that the protein turbidity phenomenon which was prevailing in natural sake at that time might have close relationship with the content of D-complex, because thereupon the turbidity producing substances had been presumed to be a certain kind of $\mathrm{N}$-containing polysaccharides. ${ }^{(3-4)}$ Though the substances are to be identified as D-comlex later, they will tentatively be mentioned as T. D. substances.

1. Decrease of D-complex by the removement of $T$. D. substances from turbid saké

Turbid saké, supplied by the courtesy of Mr. Akiyama of the Brewing Experimental Station, Tax Administration Agency, was clarified by two ways: In one, the T. D. substances were precipitated from $100 \mathrm{ml}$ turbid saké by the addition of $5 \mathrm{mg} \%$ "Clarin-S"(5), a sedimentation accelating agent composed of proteolytic enzyme preparation of Aspergillus oryzae, and in the other, the substances were centrifuged at 8000 r.p.m. for 15 minutes. Employing the original turbid saké and the clarified saké, the content of D-complex of them was compared by serological means. As seen in Table 7 , the original saké contained about $0.025 \%$ equivalent amount of D-complex, but the sake treated with "Clarin-S" contained $0.003 \%$ and the sake clarified by centrifugation contained $0.013 \%$. In parallel, the precipitate produced by the "Clarin-S" treatment was added to $100 \mathrm{ml}$ of $0.15 \mathrm{M} \mathrm{NaCl}$ solution to make up the original concentration, and the content of D-complex in the liquor was measured, resulting in a $0.013-0.025 \%$ equivalent amount of D-complex.

Table 7. The Content of D-equivalent Substances in Turbid Saké before and after Treatment

\begin{tabular}{l|c|c}
\hline \multicolumn{1}{c|}{ Samples } & Turbidity & “D " contents \\
\hline Turbid saké & W & $0.025 \%$ \\
Clarin-S treated saké & - & $0.003 \%$ \\
Centrifuged saké & \pm & $0.013 \%$ \\
Precipitate* & & $0.013-0.025 \%$
\end{tabular}

* Refer to text. 


\section{Induction of protein turbidity by the addition of D-complex}

In this experiment, sample-D was added to synthetic saké and to clear natural saké in the amount ranging from 0.0125 to $0.0500 \%$, and the presence or absence of turbidity in each concentration was observed. As it is shown in Table 8 , the sample produced turbidity in both synthetic and natural saké at the concentration of $0.025 \%$, the concentration just corresponding to that of $\mathrm{D}$-complex in turbid saké.

Table 8. Turbidity Induction Test by the Addition of D-complex

\begin{tabular}{c|c|c}
\hline $\begin{array}{c}\text { Final concentration } \\
\text { of D-complex }\end{array}$ & \multicolumn{2}{|c|}{ The degree of turbidity induced in } \\
\cline { 2 - 3 } & Synthetic saké & Natural saké \\
\hline $0.0125 \%$ & - & - \\
$0.0167 \%$ & \pm & \pm \\
$0.0250 \%$ & $H$ & + \\
$0.0500 \%$ & H \\
\hline
\end{tabular}

\section{Sedimentation of D-complex by "Clarin-S"}

In connection with the experiment mentioned above, the artificially prepared turbid saké was treated with "Clarin-S". The result was as expected succesful. The D-complex in synthetic saké was perfectly removed by the agent.

\section{Reaction between T. D. substances and anti-D-serum.}

The sample of T. D. substances prepared by centrifugation was dissolved in $0.15 \mathrm{M} \mathrm{NaCl}$ solution in an amount not sufficient to produce turbidity (about $0.025 \%$ ), and the immunological reaction between the prepared solution and the anti-D-serum was examined by one-dimensional diffusion method. The reaction was positive, indicating that $T$. D. substances are related to the D-complex in case of the application of serological means.

\section{Electrophoretic behavior of $T$. D. Substances and D-complex}

As the final evidence, electrophoretitic mobility of both $T$. D. substances and D-complex were compared by paper electrophoretic technique. The

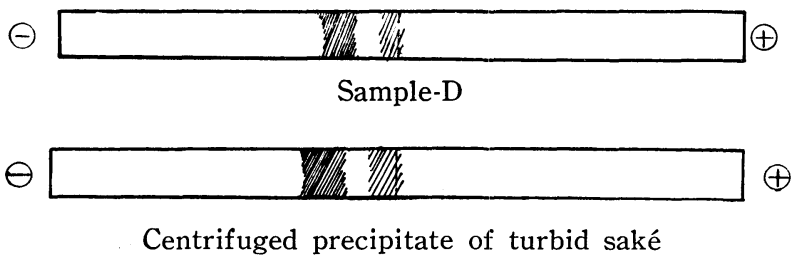

Fig. 5 Electrophoretic Behaviors of Turbidity Producing Substances Contained in Saké (on a Paper Electrophoretic Apparatus). 
results are illustrated in Figure 5. No significant difference was found among them at the following conditions: $1 / 20 \mathrm{~m}$ phosphate buffer ( $\mathrm{pH} 7.8$ ), $400 \mathrm{D}$. C. volt, 5 hours.

From the data stated in this chapter, it is doubtless that the turbidity producing substances in natural saké are identical with D-complex, and when the above substances exist in exceedingly large amounts they are the cause of turbidity.

\section{Origin of D-complex in natural saké}

As it had been found at the time of immunological studies that the koji extract contained so much of D-complex, study was directed to the components of koji, e. g., rice and Aspergillus oryzae. At first, steamed rice was saccharified by commercial diastase, but no positive reaction resulted in the serological test. Then, Aspergillus oryzae was cultivated with natural sources such as rice, wheat, and soy bean. Each of them gave positive

Table 9. Production of D-equivalent Substance in Different Media (Aspergillus oryzae, by shaking culture)

\begin{tabular}{ll|c}
\hline \multicolumn{2}{c|}{ Media } & $\begin{array}{c}\text { Content of D-equivalent } \\
\text { substance }\end{array}$ \\
\hline Rice powder medium & $0.050 \%$ \\
Henneberg-a , & 0.100 \\
Henneberg-b , & 0.006 \\
Pfeffer &,, & 0.025 \\
Czapeck &,, & 0.013 \\
Meyer &,, & 0.025 \\
Dox &,, & 0.006 \\
\hline
\end{tabular}
reaction with anti-D-serum. Furthermore, when the strain was cultivated in synthetic media by shaking or by the surface culture method, the filtrates were also proved to be positive. The results of shaking culture are shown in Table 9. As the culture in Henneberg-a medium gave the most positive result, the origin of $\mathrm{D}$ complex in natural saké may be dependent considerably on

the strain and not on the rice. Regarding the problem whether the substance is an exo-product or an endo-product of the fungus, the amount of D-equivalent substance in the filtrate and in the mycelium having been prepared from the surface culture on Pfeffer's medium was determined. As a result, no difference between the two materials observed as the amount of the D-equivalent substance was $0.050 \%$ in the mycelium and $0.050 \%$ in the filtrate.

Subsequently, the production of D-equivalent substance by different fungi was attempted. The cultivation was carried out in Pfeffer's medium by the shaking culture method. As seen in Table 10, there were a few Aspergillus strains which did not produce any D-equivalent substance. But, as a whole, the production was restricted to Aspergillus fungi, and no strain producing the substance was to be found in strains of Penicillium, Mucor, and Rhizopus.

Among the strains giving a positive result, three strains, i. e., Asp. 
Table 10. Production of D-equivalent Substance by Different Kinds of Fungi

\begin{tabular}{|c|c|c|}
\hline Strains & Growth & $\begin{array}{c}\text { Content of D-equivalent } \\
\text { substances }(\%)\end{array}$ \\
\hline A. oryzae & H & 0.050 \\
\hline A. sojae & H & 0.025 \\
\hline A. parasiticus & H & 0.006 \\
\hline A. ustus & $H$ & 0.025 \\
\hline A. versicolor & H & 0.025 \\
\hline A. fumigatus & H & 0.006 \\
\hline A. nidulans & H & 0.006 \\
\hline A. candidus & W & 0.025 \\
\hline A. clavatus & H & 0.025 \\
\hline A. wentii & H & $0.02 j$ \\
\hline A. ochraceus & Ht & 0.013 \\
\hline A. terreus & H & 0.025 \\
\hline A. tamarii & H & 0.006 \\
\hline A. flavipas & H & 0.006 \\
\hline A. inui & H & 0.000 \\
\hline A. usamii & H & 0.013 \\
\hline A. saitoi & H & 0.013 \\
\hline A. niger & W & 0.013 \\
\hline A. japonicus & H & 0.050 \\
\hline$P$. islandicum & H & 0.000 \\
\hline P. chrysogenum & H & 0.000 \\
\hline M. racemosus & + & 0.000 \\
\hline M. spinescens & + & 0.000 \\
\hline R. nigricans & W & 0.000 \\
\hline R. japonicus & $H$ & 0.000 \\
\hline R. peka & H & 0.000 \\
\hline Monascus purpureus & $H$ & 0.000 \\
\hline
\end{tabular}

oryzae, Asp. japonicus, and Asp. candidus, were cultivated in a large scale, and the presence of D-complex in the filtrate was identified by the paper electrophoretic technique.

As the summary of this chapter, the author wish to conclude that the D-complex is a special metabolic product of Aspergillus fungi, although there are some exceptional strains in which the production is doubtful.

\section{DISCUSSION}

As mentioned in the introductory chapter, the authors undertook this study with the intention to investigate the difference still existing between natural 
and artificial saké, and as well as this, by the application of recent knowledge proceeded, to improve the latter to compete with the quality of best grade natural saké.

With regard to the constituents of natural saké, a number of more than forty kinds of components have so far been elucidated, however, no paper dealing with the high molecular components has been published. Moreover, as the nature of this kind of study, any trial to reproduce natural products by the combination of well-known components has hitherto always been unsuccessful. This fact suggests that the interaction between two or more components after mixing or combination must be taken into account.

In this paper, two mucoprotein-like substances are newly reported. Though the samples themselves do not taste well, the present authors are convinced that they are playing an important role with other components in the harmony of natural saké. According to the preliminary study, the synthetic sake supplemented with the materials tasted milder than the original sake, probably owing to the buffer action. However, the authors have to confess that the quality of the prepared sake was not so fine as expected, suggesting that further studies of the components of sake and of the conditions essential for mixing or aging are necessary.

During the course of this study, the authors happened to find that the "protein turbidity" phenomenon of natural saké was closely related to the content of the above mentioned mucoproteins, and also that when they exist in excess in saké become the cause of turbidity. The most possible explanation for the occurrence of this phenomenon may be that the tendency of saké manufacturing is changing to obtain a gain in yield by adding alcohol to the fermenting broth, so that saké brewed by the application of this process may contain a larger amount of unhydrolyzed protein than the purely brewed saké.

\section{SUMMARY}

1. Two high molecular substances, D-1 and D-2 described in this paper, were isolated from natural saké and identified chemically as some kinds of mucoprotein ( $\mathrm{N}$-containing polysaccharides).

2. Preparing antiserum reacting with D-complex (D-1 and D-2), the amount of D-equivalent substance contained in commercial saké was determined serologically.

3. The protein turbidity producing substances in saké were identified as D-complex; when the content exceeded $0.015 \%$ the turbidity phenomenon occurred.

4. Most Aspergillus fungi produced D-complex even in synthetic media, but the fungi belonging to Penicillium, Mucor, and Rhizopus did not.

5. The role of D-complex in saké taste was discussed. 


\section{ACKNOWLEDGEMENT}

The authors wish to express their sincere thanks to Professor K. Sakaguchi, University of Tokyo, for his valuable advice. They are also indebted to Dr. K. Kubota, National Institute of Health of Japan, Dr. M. Yamada and Mr. Y. Akiyama, Brewing Experimental Station, Tax Administration Agency, and Mr. M. Nomoto of the Scientific Research Institute Ltd., for their kind suggestions and furnishing the samples used in this study.

\section{LITERATURES}

(1) Ando, T. And Ikeda, Y.: Bull. Agr. Chem. Soc. (Japan), 19, 221 (1955).

(2) Durrum, E. L.: J. Am. Chem. Soc., 72, 2923 (1950).

(3) INOUE, Y. AND KAWASAKI, T.: J. Soc. Brewing (Japan), 48, 431 (1953).

(4) IChikawa, K., Inoue, Y., and KaWASAKI, T.: ibid, 50, 292 (1955).

(5) Yamada, M., Akiyama, Y. Shimizu, A. And Fujil, R.: J. Agr. Chem. Soc. (Japan), 31, 127 (1957). 